\title{
Erratum to: The WHO ultrasonography protocol for assessing hepatic morbidity due to Schistosoma mansoni. Acceptance and evolution over 12 years
}

\author{
Tarik el Scheich • Martha C. Holtfreter • \\ Hendrik Ekamp • Daman D. Singh • Rodrigo Mota • \\ Christoph Hatz $\cdot$ Joachim Richter
}

Published online: 13 November 2014

(C) Springer-Verlag Berlin Heidelberg 2014

Erratum to: Parasitology Research 113 (11): 3915 - 3925 DOI 10.1007/s00436-014-4117-0

The original version of this article contained some mistake. The revised 3rd sentence in the Conclusion section is below.

Contrary to the pictorial approach, the quantitative approach by measuring the peripheral or segmental portal branch wall thickness has proved to be subject to systematical errors affecting the comparability of results between different settings and does appear not to specifically reflect schistosomal morbidity.

In Table 2 some rows have shifted: there were 3 Senegalese studies 3 of them applying the Niamey protocol on 668 patients

The online version of the original article can be found at http://dx.doi.org/ 10.1007/s00436-014-4117-0.

\section{T. el Scheich}

Department of Pediatrics, Faculty of Medicine,

Heinrich-Heine-University, Düsseldorf, FR, Germany

T. el Scheich $\cdot$ M. C. Holtfreter · D. D. Singh $\cdot$ R. Mota $\cdot$

J. Richter $(\square)$

Tropical Medicine Unit, Department for Gastroenterology,

Hepatology and Infectious Diseases, Faculty of Medicine,

Heinrich-Heine-University, Düsseldorf, FR, Germany

e-mail: Joachim.Richter@med.uni-duesseldorf.de

\section{H. Ekamp}

Department of Diagnostic Radiology, HELIOS Klinikum Wuppertal,

University of Witten/Herdecke, Witten, Germany

C. Hatz

Swiss Tropical and Public Health Institute, Basel, Switzerland

C. Hatz

Institute for Social and Preventive Medicine, University of Zürich,

Zürich, Switzerland
Table 2 Ultrasound studies on liver abnormalities due to $S$. mansoni 2001-2012

\begin{tabular}{llllr}
\hline Countries & $\begin{array}{l}\text { No. of } \\
\text { studies }\end{array}$ & $\begin{array}{l}\text { No. of } \\
\text { patients }\end{array}$ & $\begin{array}{l}\text { Niamey/all } \\
\text { US }\end{array}$ & \multicolumn{1}{c}{$(\%)$} \\
\hline Brazil & 21 & 4273 & $11 / 21$ & 52.38 \\
Kenya & 8 & 7884 & $8 / 8$ & 100.00 \\
Egypt & 5 & 7389 & $3 / 5$ & 60.00 \\
Ethiopia & 4 & 3570 & $4 / 4$ & 100.00 \\
Uganda & 4 & 3446 & $2 / 4$ & 50.00 \\
Mali & 3 & 3767 & $3 / 3$ & 50.00 \\
Sudan & 3 & 2442 & $0 / 3$ & 0.00 \\
Senegal & 3 & 668 & $3 / 3$ & 100.00 \\
Tanzania & 2 & 1804 & $2 / 2$ & 100.00 \\
Venezuela & 2 & 325 & $1 / 2$ & 50.00 \\
Madagascar & 1 & 855 & $1 / 1$ & 100.00 \\
Saudi Arabia & 1 & 174 & $0 / 1$ & 0.00 \\
Zambia & 1 & 764 & $1 / 1$ & 100.00 \\
Oman & 1 & 96 & $1 / 1$ & 100.00 \\
Italy (Madagascan & 1 & 7 & $1 / 1$ & 100.00 \\
$\quad$ patients) & & & & 68.33 \\
Total & 60 & 37,424 & $41 / 60$ & \\
\hline
\end{tabular}

US ultrasound methods 